\title{
WCTFR : WRAPPING CURVELET TRANSFORM BASED FACE RECOGNITION
}

\author{
Arunalatha J S ${ }^{1}$, Ramachandra A C ${ }^{2}$, Tejaswi $V^{3}$, Shaila $\mathrm{K}^{1}$, Raja K B ${ }^{1}$, \\ Dinesh Anvekar ${ }^{4}$, Venugopal K R ${ }^{1}$, S S Iyengar ${ }^{5}$, L M Patnaik ${ }^{6}$ \\ ${ }^{1}$ Department of Computer Science and Engineering University Visvesvaraya \\ College of Engineering, Bangalore University, Bangalore-560001 \\ ${ }^{2}$ Alpha College of Engineering, Bangalore \\ ${ }^{3}$ National Institute of Technology, Surathkal, Karnataka \\ ${ }^{4}$ Nitte Meenakshi Institute of Technology, Bangalore. \\ ${ }^{5}$ Florida International University, USA \\ ${ }^{6}$ Indian Institute of Science, Bangalore, India
}

\begin{abstract}
The recognition of a person based on biological features are efficient compared with traditional knowledge based recognition system. In this paper we propose Wrapping Curvelet Transform based Face Recognition (WCTFR). The Wrapping Curvelet Transform (WCT) is applied on face images of database and test images to derive coefficients. The obtained coefficient matrix is rearranged to form WCT features of each image. The test image WCT features are compared with database images using Euclidean Distance (ED) to compute Equal Error Rate (EER) and True Success Rate (TSR). The proposed algorithm with WCT performs better than Curvelet Transform algorithms used in [1], [10] and [11].
\end{abstract}

\section{KEYWORDS}

Biometrics, Curvelet, Total Success Rate, Euclidean Distance.

\section{INTRODUCTION}

The biological features are inherent to physiological and behavioral features of the person. Features such as Fingerprint, Iris, and Face are physiological in nature and remain constant throughout. The Keystroke and Signature are behavioral in nature which may not remain constant. Compared to all biological features, Face recognition technology has been considered as one of the solutions for high security systems and has a great deal of interest both in academics and industry. Though face recognition is physiological in nature the feature extracted may not be constant and challenged by variations in illumination, pose, and occlusion. The facial expressions are also used for communicating emotions, disagreement and intensions.

There are various challenges in designing an algorithm for face recognition, some of the common ones are aging problem, small interclass differences, changes in expressions, changes in positions, illumination etc.. Feature extraction is one of the techniques which can be used to solve these Dhinaharan Nagamalai et al. (Eds) : CSITA, ARIN, ISPR - 2015 
problems. There are two ways to extract the features of the face, they are complete subspace analysis and local feature description.

Better features can be extracted using Curvelet theory than by Wavelet mechanism. Curvelet theory has powerful frame work to extract key local facial features which includes multi scale and multi direction. The features of test biometric are compared with features of each biometric samples of an enrolment module using either distance formulae or classifiers to authenticate validity of a person.

Motivation: Face recognition does not require cooperation of a person and can be captured at reasonable distance. Face recognition is a challenging task to recognize due various parameters such as illumination, pose, expression, background, eyeglasses, makeup etc.., and hence robust algorithm is necessary to recognize the face under the variation of these parameters to make the biometric technology card-less, paperless and cashless.

Contribution: In this paper, WCTFR algorithm is proposed to recognize a person. The features of test set and database are obtained using wrapping curvelet transform. The obtained coefficients are rearranged to get feature set of database and test set. The obtained features are compared using Euclidean Distance to compute EER and TSR.

Organization: This paper is organized into following sections. Section 2 is an overview of related work. Algorithm for proposed system is given in section 3. Performance Analysis of the system is presented in Section 4 and Conclusions are contained in Section 5.

\section{RELATED WORK}

Elaiwat et al., [1] present a Curvelet-based feature extraction algorithm for 3-D face recognition. This identifies important key-points such as scale, orientation, curvelet position, spatial position and magnitude on the face by examining curvelet coefficients in each subband. Rotation-invariant multi-scale local surface descriptors are built around the identified key points to extract distinctive facial features for robust feature matching. The proposed algorithm is robust and accurate for varying illumination and facial expressions with a better verification rate than [2], [3], [4] and [5].

Hui and $\mathrm{Hu}[6]$ proposed an algorithm for face recognition. Compared to wavelet transforms, the curvelet transform has better directional and edge representation abilities; hence the face image is decomposed to get low frequency coefficients by curvelet transform. (2D) $)^{2}$ PCA with an exponential decay factor is applied on these selected coefficients to extract feature vectors and thus achieves reduction in dimension. The nearest neighbor classifier is adopted for classification. Experiment is carried out on ORL and Yale face database.

Muzhir and Sulaiman [7] presented a feature extraction approach using wavelet-curvelet technique in order to extract facial features. This approach is based on the similarities preserved by wavelet and curvelet transform. This technique aims to reduce the dimensionality to reduce the computational power and memory consumption, the nearest mean classifier is adopted to recognize different faces.

Radha and Nallammal [8] presented a comparative analysis of face recognition methods using PCA, Linear discriminant analysis and Independent Component analysis based on curvelet 
transform. Curvelet transform is multi-resolution analysis method to improve directional elements with an isotropy and better ability to represent sparse edges and other singularities along curves. These approaches are used for dimensionality reduction. The LDA based curvelet transform gives a better recognition rate and efficient dimensionality reduction technique compared to other two methods.

Shafin et al., [9] proposed a face recognition method that uses curvelet texture features for face representation. Features are computed by taking mean and standard deviation of transformed face images and captures more accurate edge information which improves performance and reduces complexity of computation. The system is evaluated with Yale, ORL and Face94 databases. Ashirbani and Jonathm [10], describes the recognition of facial expression from still images using combination of digital curvelet transform and local binary patterns. The curvelet transform is applied to face at specific scale and orientation then from selected curvelet sub-bands local binary patterns are extracted to form the descriptive feature set of expressions. The expression recognition is performed using a nearest neighbor classifier with chi-square as dissimilarity metric.

\section{ALGORITHM}

Problem definition: The Wrapping Curvelet Transform Technique is applied on a set of face test image and a set of training images of a face database.

The objectives are to:

(i) Increase the Total Success Rate (TSR) and

(ii) Reduce the Equal Error Rate (EER).

Algorithm of proposed face recognition system is as given in Table 1.

The face images are first read from the ORL database. Preprocessing is performed to resize the image into $256 \times 256$ pixels. The color images are then converted to gray scale image. The Wrapping Curvelet Transform is applied on to the process test model at scale 5 and angle 16. The above procedure is applied for each test image features. The Euclidean distance parameter are considered with the test image and compared with the database image features. The Euclidean distance is less than the threshold value between the test image and the database image, then the image is considered to have match with the database image.

Table 1: WCTFR: Wrapping Curvelet Transform based Face Recognition.






\section{PERFORMANCE ANALYSIS}

Table 2: FAR, FRR and TSR for different PIDB and PODB

\begin{tabular}{|c|c|c|c|c|c|c|c|c|c|c|c|c|c|c|c|}
\hline \multirow{3}{*}{ Threshold } & \multicolumn{15}{|c|}{ PIDB:PODB } \\
\hline & \multicolumn{3}{|c|}{$5: 10$} & \multicolumn{3}{|c|}{$5: 15$} & \multicolumn{3}{|c|}{$5: 5$} & \multicolumn{3}{|c|}{$10: 5$} & \multicolumn{3}{|c|}{$15: 5$} \\
\hline & FRR & FAR & TSR & FRR & FAR & TSR & FRR & FAR & TSR & FRR & FAR & TSR & FRR & FAR & 16 \\
\hline 3.0 & 1.00 & 0.00 & 0.00 & 1.00 & 0.00 & 0.00 & 1.00 & 0.00 & 0.00 & 0.100 & 0.00 & 0.00 & 0.93 & 0.00 & 00 \\
\hline 4.4 & 0.80 & 0.00 & 0.20 & 0.80 & 0.00 & 0.20 & 0.80 & 0.00 & 0.20 & 0.90 & 0.00 & 0.10 & 0.86 & 0.00 & 0.6 \\
\hline 5.6 & 0.60 & 0.00 & 0.40 & 0.60 & 0.00 & 0.40 & 0.60 & 0.00 & 0.40 & 0.80 & 0.00 & 0.20 & 0.80 & 0.00 & 0.13 \\
\hline 6.2 & 0.60 & 0.00 & 0.40 & 0.60 & 0.00 & 0.40 & 0.60 & 0.00 & 0.40 & 0.70 & 0.00 & 0.30 & 0.73 & 0.00 & 0.20 \\
\hline 6.3 & 0.40 & 0.00 & 0.60 & 0.40 & 0.00 & 0.60 & 0.40 & 0.00 & 0.60 & 0.60 & 0.00 & 0.40 & 0.66 & 0.00 & 0.26 \\
\hline 6.6 & 0.40 & 0.00 & 0.60 & 0.40 & 0.00 & 0.60 & 0.40 & 0.00 & 0.60 & 0.50 & 0.00 & 050 & 0.60 & 0.00 & 0.33 \\
\hline 6.8 & 0.40 & 0.00 & 0.60 & 0.40 & 0.00 & 0.60 & 0.40 & 0.00 & 0.60 & 0.50 & 0.00 & 0.50 & 0.53 & 0.00 & 0.40 \\
\hline 7.3 & 0.40 & 0.00 & 0.60 & 0.40 & 0.00 & 0.60 & 0.40 & 0.00 & 0.60 & 0.50 & 0.00 & 0.50 & 0.40 & 0.00 & 0.46 \\
\hline 7.7 & 0.20 & 0.00 & 0.80 & 0.20 & 0.00 & 0.80 & 0.20 & 0.00 & 0.80 & 0.40 & 0.00 & 0.60 & 0.33 & 0.00 & 0.53 \\
\hline 7.9 & 0.20 & 0.00 & 0.80 & 0.20 & 0.00 & 0.80 & 0.20 & 0.00 & 0.80 & 0.20 & 0.00 & 0.80 & 0.20 & 0.00 & 0.66 \\
\hline 8.9 & 0.20 & 0.00 & 0.80 & 0.20 & 0.06 & 0.80 & 0.20 & 0.00 & 0.80 & 0.20 & 0.00 & 0.80 & 0.13 & 0.13 & 0.73 \\
\hline 9.5 & 0.20 & 0.00 & 0.80 & 0.20 & 0.06 & 0.80 & 0.20 & 0.00 & 0.80 & 0.10 & 0.10 & 0.90 & 0.13 & 0.20 & 0.73 \\
\hline 9.6 & 0.00 & 0.00 & 0.99 & 0.00 & 0.06 & 0.99 & 0.00 & 0.00 & 0.99 & 0.10 & 0.20 & 0.90 & 0.06 & 0.20 & 0.80 \\
\hline 10.1 & 0.00 & 0.10 & 0.99 & 0.00 & 0.13 & 0.99 & 0.00 & 0.00 & 0.99 & 0.10 & 0.40 & 0.90 & 0.06 & 0.40 & 0.80 \\
\hline 10.3 & 0.00 & 0.40 & 0.99 & 0.00 & 0.40 & 0.99 & 0.00 & 0.20 & 0.99 & 0.10 & 0.80 & 0.90 & 0.06 & 0.60 & 0.80 \\
\hline 11.2 & 0.00 & 0.50 & 0.99 & 0.00 & 0.60 & 0.99 & 0.00 & 0.40 & 0.99 & 0.10 & 0.80 & 0.99 & 0.00 & 1.00 & 0.86 \\
\hline 12.0 & 0.00 & 0.60 & 0.99 & 0.00 & 0.73 & 0.99 & 0.00 & 0.40 & 0.99 & 0.00 & 0.80 & 0.99 & 0.00 & 1.00 & 0.86 \\
\hline
\end{tabular}

The Performance Parameters such as FRR, FAR, EER and TSR for the ORL face database is discussed in detail for the proposed model. The recognition rate for different number of features per image is calculated using Euclidean distance. The performance parameters are computed for different number of Persons in Database (PID) and Persons out of Database (POD). We have considered PID: POD as 5:10, 5:15, 5:5, 10:5 and 15:5. The variations of FRR, FAR and TSR with the threshold for ORL database is given in Table 2. The values of FAR and TSR increases from 0 to maximum value as threshold value increases. The values of percentage FRR decreases from 100 to 0 as threshold value increases. The maximum success rate of the proposed algorithm for ORL database is $99.7 \%$. The values of FRR and FAR decreases and increases respectively as threshold value increases. It is observed that the value of EER is zero for the threshold value of 10 with the Percentage of TSR is 99.7.

Table 3 shows the percentage variation of TSR and EER of the proposed model for different number of Persons Inside Database (PID) and Persons Outside Database (POD). It is observed from the table, when number of PID is low TSR is high and EER is low. When number of PID is high, TSR is low and EER is high. It is also observed that EER is low, at lower threshold values as we increase the number of persons inside database. When PID is almost equal to POD, the probability of genuine samples being accepted and invalid samples being rejected is high, hence TSR is 0.997 . 
Table 3: The variation of TSR and EER for different PID:POD ratios

\begin{tabular}{|c|c|c|}
\hline PID:POD & TSR & EER \\
\hline $5: 10$ & 1.00 & 0.00 \\
\hline $5: 15$ & 0.95 & 0.50 \\
\hline $5: 5$ & 1.00 & 0.00 \\
\hline $10: 5$ & 0.90 & 0.10 \\
\hline $15: 5$ & 0.86 & 0.13 \\
\hline
\end{tabular}

Table 4: Comparison of TSR(\%) of proposed WCTFR with [1], [10] and [11].

\begin{tabular}{|c|c|c|c|}
\hline $\begin{array}{c}\text { S1 } \\
\text { No }\end{array}$ & Authors & Techniques & TSR(\%) \\
\hline 1 & Huo and Song [11] & $\begin{array}{c}\text { Curvelet } \\
(3,8)+\text { SPCA }\end{array}$ & 93.70 \\
\hline 2 & $\begin{array}{c}\text { Saha and Jonathan } \\
{[10]}\end{array}$ & $\begin{array}{c}\text { Curvelet } \\
(3,8)+\text { LBP }\end{array}$ & 93.69 \\
\hline 3 & Elaiwat et al.,[1] & $\begin{array}{c}\text { Curvelet } \\
(2,3) \\
\text { +3D Images }\end{array}$ & 98.20 \\
\hline 4 & $\begin{array}{c}\text { Proposed } \\
\text { algorithm } \\
\text { (WCTFR) }\end{array}$ & $\begin{array}{c}\text { Wrapping } \\
\text { Curvelet (5, } \\
\mathbf{1 6 )}\end{array}$ & 99.70 \\
\hline
\end{tabular}

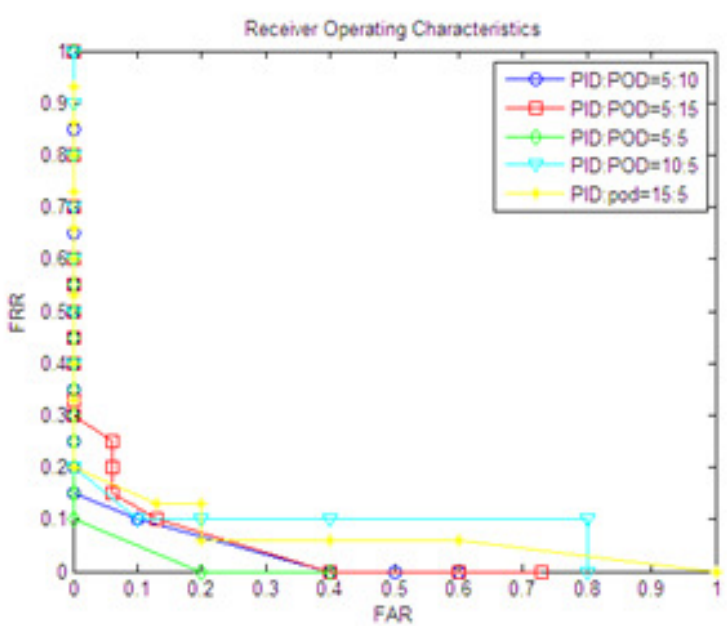

Fig. 1: ROC characteristics of WCTFR.

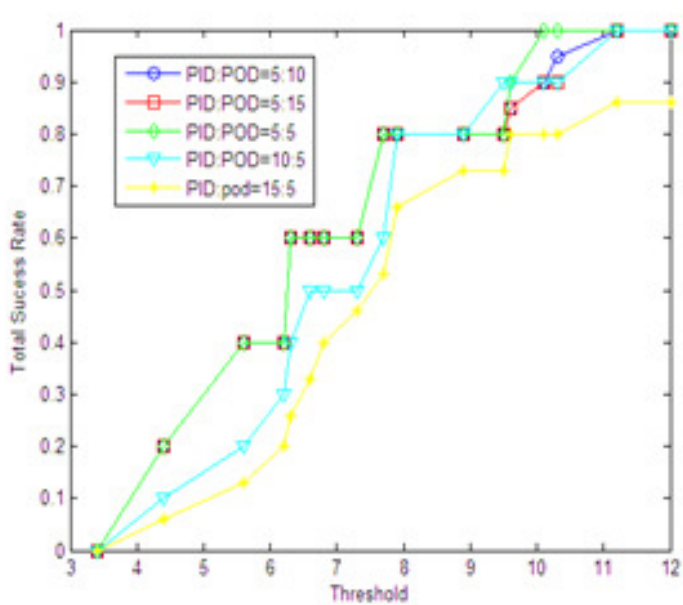

Fig. 2: Total Success Rate versus Threshold for different PID:POD ratios for WCTFR. 
The Receiver Operating Characteristics (ROC) for different PID: POD ratios are as shown in Fig.1, it is a plot of FRR versus FAR for different PID: POD ratios. The plot of TSR versus Threshold for different PID: POD ratios are as shown in Fig. 2. It is observed that TSR is high for lower threshold values as number of persons outside database increases and TSR is low for higher threshold values as number of persons inside database increases. The proposed algorithm gives maximum TSR of 0.997 when it is operated between thresholds of 10.5 to 12.0 of feature set. The Performance of Proposed method is compared with the existing algorithms which are given in Table 4. In the existing algorithms, they have used Curvelet with level three and orientation eight and also they have used additional techniques like SPCA and LBP. In the proposed WCTFR algorithm we have considered level five and orientation sixteen which increases the directional features and hence the algorithm has better performance than [1], [10] and [11] by $1.5 \%$.

\section{CONCLUSIONS}

The face recognition is a Physiological biometric trait to identify a person efficiently. In this paper Wrapping Curvelet Transform based Face Recognition algorithm is proposed. The curvelet Coefficients are generated from face images and resized to Coefficient Matrix to form features. The EER and TSR are computed by comparing a feature of test image with database image features using Euclidean Distance. It is observed that performance parameter values of EER and TSR are better in the case of proposed algorithm compared with the existing algorithms.

\section{REFERENCES}

[1] S. Elaiwat, M. Bennanoun, F. Boussaid and A. El-sallam, "3-D Face Recognition using Curvelet Local Features," IEEE Signal Processing Letters, vol. 21, no. 2,pp. 172-175, February 2014.

[2] P. Liu, Y. Wang, D. Huang, Z. Zhang, and L. Chen, "Learning the Spherical Harmonic Features for 3-D Face Recognition," IEEE Transactions on Image Processing, vol. 22, no. 3, 2013.

[3] D Smeets, J Keustermans, D Vandermeulen, and P Suetens, "Meshsift: Local Surface Features for 3D Face Recognition under Expression Variations and Partial Data," Computer Vision and Image Understanding, vol. 117, no. 2, 2013.

[4] Y. Ming and Q. Ruan, "Robust Sparse Bounding Sphere for 3-D Face Recognition," Image Vision and Computation, vol. 30, no. 8, 2012.

[5] Y. Lei, M. Bennamoun, M. Hayat, and Y. Guo, "An Efficient 3-D Face Recognition Approach using Local Geometrical Signatures," Pattern Recognition, 2013.

[6] Ma Hui and Hu Fengsong, "Face Recognition using Curvelet Transform and (2D) ${ }^{2} \mathrm{PCA}$," International Journal on Education and Management Engineering, pp. 23-28, 2012.

[7] Muzhir Shaban Al-Ani1 and Alaa Sulaiman Al-waisy, "Face Recognition Approach based on Wavelet - Curvelet Technique," International Journal on Signal and Image Processing, vol. 3, no. 2, pp. 21-31, 2012.

[8] V Radha and N Nallammal, "Comparative Analysis of Curvelets Based Face Recognition Methods," Proceedings of the World Congress on Engineering and Computer Science, vol. 1, 2011. 
[9] Shafin Rahman, Sheikh Motahar Naim, Abdullah Al Farooq and Md Monirul Islam, "Curvelet Texture based Face Recognition using Principal Component Analysis," International Conference on Computer and Information Technology, pp. 45-50, 2010.

[10] Ashirbani Saha and Q. M. Jonathan Wu, "Facial Expression Recognition using Curvelet based Local Binary Patterns," IEEE International Conference on Acoustics Speech and Signal Processing, pp. 2470-2473, 2010.

[11] Hanjun Huo and Enuo Song, "Face Recognition using Curvelet and Selective PCA,"International Conference on Intelligent Control and Information Processing, Dalian, China, pp. 348-351, 2010. 